\title{
Commentaries
}

\section{Helicobacter pylori resists arrest}

Testing for the presence of Helicobacter pylori and subsequent eradication of the infection is a key aspect in the management of dyspepsia, which is now the largest single area of cost for primary care in the United Kingdom. ${ }^{1}$ Since the bacterium was discovered in 1982 and its role in the aetiology of peptic ulcer disease became established, significant advances have been achieved in antimicrobial treatment with current regimens enabling successful eradication in 85 to $90 \%$ of patients. ${ }^{23}$ The reasons for treatment failure in the remaining small but significant group of infected individuals is often not clearly established but resistance to metronidazole and to clarithromycin are generally considered to be the primary factors. Both of these antibiotics are widely used in current regimens for eradicating $H$ pylori although rates of resistance vary significantly from 10 to $50 \%$ for metronidazole, and from 0 to $15 \%$ for clarithromycin, depending on the population group surveyed. ${ }^{4}$ For instance, in a recent study of dyspeptics attending an open access endoscopy clinic in mid-Essex, we found pretreatment rates of $6 \%$ for clarithromycin and $37 \%$ for metronidazole (L Teare, personal communication). The fact that resistance rates for clarithromycin may be gradually increasing is particularly worrying as it is a most useful antibiotic against $H$ pylori because of its absorption and stability properties in the stomach. Consequently it is a key component in eradication regimens, particularly those for first line treatment in areas of high prevalence of metronidazole resistance. ${ }^{3}$

The mode of action of clarithromycin after penetrating the cell wall is to bind to ribosomes and disrupt protein synthesis. Decrease in the binding of clarithromycin to the ribosome is linked to the development of resistance which in turn is attributed to various point mutations in the two 23S rRNA genes of $H$ pylori. These mutations were first reported in US isolates in 1996 by Versalovic and colleagues $^{5}$ and since then have been confirmed in clinical isolates from France, ${ }^{4}$ Sweden, ${ }^{6}$ Canada, ${ }^{7}$ and the Netherlands. ${ }^{8}$ The two most common mutations are those in which adenine residues are replaced by guanine at positions 2143 and 2144 (transition mutations A2143G and A2144G equivalent to Escherichia coli coordinates 2058 and 2059) although other rarer mutations may occur (e.g. A2116G and A2143C). Detection of the two main mutations were first based on the use of PCR assays combined with digestion with specific restriction endonucleases. Such molecular tests were advocated for speed as they could be completed on cultures within 24 hours whereas conventional microbiological culture based susceptibility testing (E-test or disc diffusion) requires up to four days. Subsequent technical developments have led to the availability of more rapid mutant specific probe based assays, namely a PCR-oligonucleotide ligation assay ${ }^{9}$ and a DNA enzyme linked immunoassay (DEIA). ${ }^{10}$ The optimal conditions were defined for each probe which discriminated single base variations and a complete correlation with culture methods was found.

Marais et al, in this issue (see page 463), have described a further novel development to the DEIA technique, in which mutant specific probes were used in a rapid (one day) laboratory assay that could be applied directly to gastric biopsy samples. This assay tested both for the presence of $H$ pylori and for evidence of resistance to clarithromycin, but most importantly without the need for culture. The technique has the potential of providing a result within 24 hours whereas conventional culture from biopsy samples takes up to five days with a further three to four days for susceptibility testing. The method, which was based on the use of a colorimetric hybridisation assay with four probes-for the wild (sensitive) type and three resistant mutants-offered a significant improvement in reporting time as the result could be available within a day of endoscopy. When tested on 33 biopsy samples infected with resistant strains of $H$ pylori, most mutations were either at A2144G or A2143G with a few at A2143C. For some samples, the results highlighted the presence of multiple genotypes with the A2143C mutation mostly present either with the wild type or with the A2143G mutation. These results were interpreted as possibly mixtures of strain types in the sample or as heterozygotic with respect to the two 23S rRNA gene copies.

The use of DNA amplification or probing technology, or both, in antibiotic resistance gene detection is a developing area of clinical bacteriology with already recognised applications for detection of resistance in Staphylococcus aureus (MRSA) and in Mycobacterium tuberculosis. The work of Marais and colleagues demonstrates how their time-saving and potentially cost effective approach can be applied to $H$ pylori. It will be interesting to see to what extent this approach finds a place in the routine diagnostic laboratory, particularly as definitive therapy for $H$ pylori depends on information about metronidazole as well as clarithromycin susceptibilities. At present, no DNA based assay is available for detecting metronidazole resistance, and as resistance to that antibiotic is widespread, susceptibility results are essential for prescribing.

In conclusion, this is an interesting new technical development, although in the short term it would seem unlikely to replace the need for culture based sensitivity testing in the clinical laboratory given the relatively sophisticated technical aspects involved combined with the need to establish rigorous quality indicators to satisfy accreditation requirements. At present there is no information on such mutations in strains isolated from UK patients, so the reliability of the DNA assay would also need to be assessed rigorously on local populations in view of the recognised genomic diversity of $H$ pylori.

R J OWEN

Laboratory of Enteric Pathogens,

Central Public Health Laboratory,

Colindale Avenue, London NW9 5HT, UK

1 Delaney RC, Hobbs FDR. Helicobacter pylori eradication in primary care. $B M F$ 1998;316:1634.

Harris A. Current regimens for treatment of Helicobacter pylori infections. Br Med Bull 1998;54:195-206.

3 Misiewiez A, Harris AW, Bardhan KD, et al. One week triple therapy for Helicobacter pylori: a multicentre comparative study. Gut 1997;41:735-9.

4 Megraud F. Antibiotic resistance in Helicobacter pylori infections. $\mathrm{Br}$ Med Bull 1998;54:207-16.

5 Versalovic J, Shortridge D, Kibler K, et al. Mutations in 23S rRNA are associated with clarithromycin resistance in H. pylori. Antimicrob Agents Chemother $1996 ; 40: 477-80$

6 Hulten K, Gibreel A, Skold O, et al. Macrolide resistance in H. pylori: mechanism and stability in strains from clarithromycin-treated patients. Antimicrob Agents Chemother 1997;41:2550-3. 
7 Taylor DE, Zhongming GE, Purych D, et al. Cloning and sequence analysis of two copies of a $23 \mathrm{~S}$ rRNA gene from $\mathrm{H}$. pylori and association of clarihromycin resistance with $23 \mathrm{~S}$ rRNA mutations. Antimicrob Agents Chemother 1997;41:2621-8.

8 Debets-Ossenkopp. YJ, Sparrius M, Kusters JJ, et al. Mechanism of clarithromycin resistance in clinical isolates of Helicobacter pylori. FEMS Microbiol Lett 1996;142:37-42.
9 Stone GG, Shortridge D, Versalovic J, et al. A PCR-oligonucleotide ligation assay to determine the prevalence of $23 \mathrm{~S}$ rRNA gene mutations in clarithromycin resistant $\mathrm{H}$. pylori. Antimicrob Agents Chemother 1997;41: $712-14$

10 Pina M, Occhialini A, Monteiro L, et al. Detection of point mutations associated with resistance of Helicobacter pylori to clarithromycin by hybridization in liquid phase. F Clin Microbiol 1998;36:3285-90.

\section{Omeprazole: Helicobacter pylori makes thee greater yet}

Helicobactoer pylori affects the magnitude of acid inhibition by proton pump inhibitors..$^{1-3}$ In an early study, omeprazole produced greater acid suppression in subjects with $H$ pylori infection than in those without infection. ${ }^{1}$ Direct evidence of the interaction between $H$ pylori infection and the efficacy of omeprazole came from studies in which cure of the infection resulted in a decrease in the antisecretory effect exerted by omeprazole, both in healthy subjects ${ }^{2}$ and in patients with duodenal ulcer disease. ${ }^{3}$

In this issue (see page 468) Gillen et al confirm the effect of $H$ pylori on the reduction in acidity produced by omeprazole. The authors have tried to elucidate the mechanism responsible for this effect. They report data which seem to refute the previously postulated hypothesis ${ }^{1-3}$ that ammonia is important in the interaction between $H$ pylori and omeprazole.

Nevertheless, there are many good reasons favouring the ammonia buffering system hypothesis. Firstly, this theory takes into account the equilibrium between secreted protons and ammonia produced by $H$ pylori. The ammonia buffering system has a minimal apparent effect in the absence of omeprazole therapy when the $\mathrm{H}^{+}$concentrations in the stomach are high, but it becomes effective when $\mathrm{H}^{+}$concentrations decrease in response to omeprazole treatment. ${ }^{1-4}$ Secondly, the loss of effectiveness of secretory inhibitors after eradication of the organism, along with the disappearance of ammonia from the stomach, further support this theory..$^{2-5}$ Thirdly, the ammonia hypothesis could explain why this effect is less pronounced with $\mathrm{H}_{2}$ receptor antagonists. As acid suppression achieved using $\mathrm{H}_{2}$ receptor antagonists is lower than that with proton pump inhibitors, ammonia cannot influence intragastric $\mathrm{pH} .{ }^{5}$ Fourthly, ammonia produced by $H$ pylori may increase $\mathrm{H}^{+}$back-diffusion across damaged gastric mucosa, thus lowering $\mathrm{H}^{+}$concentrations in the stomach. $^{5}$ The effect of the ammonia buffering system would become particularly apparent with a further decrease in $\mathrm{H}^{+}$concentrations induced by omeprazole therapy. Finally, the mechanisms whereby $H$ pylori and omeprazole interact may be dependent upon dose and duration of antisecretory therapy. In earlier studies, measurements were conducted after a one week course of omeprazole $20 \mathrm{mg}$ daily. ${ }^{1-4}$ In Gillen et al's study, a higher dose of omeprazole (40 mg o.d.) was administered for eight weeks. With this treatment, in contrast to low dose, short term therapy, $H$ pylori infection is suppressed and ammonia production is therefore relatively low. Overall, the enhanced antisecretory effect of short term omeprazole therapy in $H$ pylori infected subjects may be a result of the production of acid neutralising compounds by the bacterium.

Gillen et al propose an alternative mechanism for the greater $\mathrm{pH}$ raising effect of omeprazole therapy during $H$ pylori infection. They postulate that the severity of $H$ pylori induced corpus gastritis is exacerbated by omeprazole; this would lead to decreased acid secretion and increased $\mathrm{pH}$. Unfortunately, no data to support this proposal are provided. Gastritis was not assessed. Furthermore, the gastritis hypothesis does not fully explain the increased intragastric $\mathrm{pH}$ observed during potent antisecretory therapy in subjects with $H$ pylori infection. Firstly, the combination of high grade body gastritis and hyposecretion does not occur in patients with duodenal ulcer disease. ${ }^{3}$ These patients, as the authors themselves point out, are acid hypersecretors. ${ }^{7}$ Secondly, the increased efficacy of antisecretory therapy has been observed on the first day of administration of a reversible proton pump inhibitor (BY 841) to asymptomatic $H$ pylori positive subjects. ${ }^{8}$ It is highly unlikely that redistribution of $H$ pylori to the gastric corpus and subsequent worsening of body gastritis occurs within a few hours of administration of acid inhibitory therapy. Finally, the decrease in response to omeprazole after eradication of $H$ pylori is rapid and does not progress, whereas gastritis continues to improve during the first few months after eradication. ${ }^{9}$ Thus this mechanism is speculative.

Some drawbacks in Gillen et al's study mitigate against the validity of the gastritis hypothesis. Firstly, the diagnosis of $H$ pylori status was based on urea $-{ }^{14} \mathrm{C}$ breath tests alone. No single technique, however, can be considered ideal for the detection of $H$ pylori infection. The high $\mathrm{pH}$ and ammonia values observed by Gillen et al in breath test negative subjects (fig 1) are, in our view, suggestive that these subjects were, in fact, $H$ pylori positive. Serological or histological analysis should have been conducted in these subjects. Secondly, gastric acid output studies were performed before and after omeprazole administration to separate groups of $H$ pylori positive and negative subjects, instead of studying subjects before and after cure of the infection. Thirdly, gastric acid secretion and $\mathrm{pH}$ were measured in single samples of gastric juice obtained during 30 minute periods. Consequently, hourly variations in gastric secretion and ammonia output, additional factors such as reflux of duodenal alkali into the stomach, and loss of gastric contents into the duodenum were not evaluated. In previous studies, this has been overcome by prolonged overnight measurements and by perfusing the stomach with volume markers. ${ }^{4}$ The question therefore arises whether the values obtained by Gillen et al are representative of acid secretion of the subjects studied. Fourthly, the role of other neutralising substances with a pKa above $\mathrm{pH} 7$ such as secreted immunoglobulins and albumin leaking through a damaged mucosa was not evaluated. ${ }^{10}$ Finally, statistical analysis of the data is difficult to understand. Twenty $\mathrm{H}$ pylori negative and $12 \mathrm{H}$ pylori positive subjects were included in the study. Ammonia samples of a number of subjects are reported to be "not available". We wonder whether the authors have included only the subjects with available ammonia values in their comparisons of group medians (acid and ammonia).

In conclusion, Gillen et al's study confirms the previous observations that acid suppressive treatment in subjects with $H$ pylori infection is more effective than in $H$ pylori negative subjects. ${ }^{1-5} 9$ In addition, the authors show that this effect persists after long term treatment with proton pump inhibitors. However, the mechanism underlying the $\mathrm{pH}$ elevating effect of omeprazole therapy in $H$ pylori positive subjects is not elucidated and awaits further study.

D PANTOFLICKOVA A L BLUM 
Division of Gastroenterology,

Department of Medicine,

University Hospital,

CHUV, MAT - anc. CI,

CH-1011, Lausanne, Switzerland

Intestinal Diseases Research Programme,

McMaster University,

Hamilon,

Ontario, Canada

Correspondence to: Dr Pantoflickova (email: dpantofl@hola.hospvd.ch).

1 Verdú EF, Armstrong D, Fraser R, et al. Effect of Helicobacter pylori status on intragastric $\mathrm{pH}$ during treatment with omeprazole. Gut 1995;36:539-43.

2 Verdú EF, Armstrong D, Idström J-P, et al. Effect of curing Helicobacter pylori infection on intragastric $\mathrm{pH}$ during treatment with omeprazole. Gut 1995;37:743-8.

3 Labenz J, Tillenburg B, Peitz U, et al. Helicobacter pylori augments the $\mathrm{pH}$-increasing effect of omeprazole in patients with duodenal ulcer. Gastroenterology 1996;110:725-32.
4 Bercik P, Verdú EF, Armstrong D, et al. H. pylori-related increase in omeprazole (OME) effect is associated with ammonia production [abstract]. Gastroenterology 1996;110:A64.

5 van Herwaarden MA, Samsom M, Van Nispen CHM, et al. H. pylori eradication reduces the effect of lansoprazole but not that of ranitidine on intra-

gastric $\mathrm{pH}$ [abstract]. Gastroenterology 1998;114:A321.
P BERCIK 6 Desai MA, Vadgama PM. Enhanced $\mathrm{H}^{+}$diffusion by $\mathrm{NH}_{4}^{+} / \mathrm{HCO}_{3}^{-}$: implications for H. pylori associated peptic ulceration. Digestion 1993;54: implica.

7 Gillen D, el-Omar EM, Wirz AA, et al. The acid response to gastrin distinguishes duodenal ulcer patients from Helicobacter pylori-infected healthy subjects. Gastroenterology 1998;114:50-7.

8 Riedel T, Hartmann M, Lühmann R, et al. The reversible H/K ATP-ase antagonist BY 841 is more effective in elevating gastric $\mathrm{pH}$ in Helicobacter pylori positive than in $\mathrm{Hp}$ negative volunteers [abstract]. Gastroenterology pylori positive than

9 Verdú EF, Armstrong D, Idström JP, et al. Intragastric $\mathrm{pH}$ during treatment with omeprazole: role of Helicobacter pylori and H. pylori-associated gastritis. Scand f Gastroenterol 1996;31:1151-6.

10 Makhlouf GM, Blum AL, Moore EW. Undissociated acidity of human gastric juice. Measurement and relationship to protein buffers. Gastroenterology 1970;58:345-51.

\section{The hiatus hernia slides back into prominence}

The report on hiatus hernia by Kahrilas et al in this issue (see page 476) represents a milestone in the investigation of the impact of hiatus hernia on the mechanics of the gastrooesophageal junction. Casual readers will probably not appreciate this - their first reaction more likely being that there is nothing new in this report. This reaction is understandable as Kahrilas et al's findings are what would be predicted. The study is a milestone because it has actually made real in vivo measurements in humans. These measurements give a sound foundation for studies which will undoubtedly follow in the future and which will probe how hiatus hernia influences the mechanics of individual episodes of gastro-oesophageal reflux.

The casual reader is also unlikely to appreciate fully the technical demands presented by the measurements made by Kahrilas et al which is, of course, why there has been so much theory and deduction and so little direct observation in this field. Interpretation of manometry and fluoroscopy relied on spatial referencing of major soft tissue landmarks which was provided by the novel "tags" of the radioopaque clips. Performance of high quality pull-through manometry simultaneously with fluoroscopy, and its accurate synchronisation is also a significant task. The biggest challenge though, was to analyse and condense the imaging and pressure data in order to show accurately located axial and radial patterns of pressure across the diaphragmatic hiatus, hernial pouch and lower oesophageal sphincter.

Over this century, there have been widely differing views and major swings of mainstream opinion about whether, and if so how, hiatus hernia impairs gastro-oesophageal competence. ${ }^{12}$ There are two main reasons for this lack of agreement. Firstly, there have been very few actual data, given the measurement difficulties. Secondly, thinking about mechanisms of gastro-oesophageal reflux has been too simplistic. As Kahrilas et al indicate, various mechanisms have been championed as the cause of gastrooesophageal reflux, such as hiatus hernia, loss of the angle of His, failure of extrinsic diaphragmatic compression of the oesophagus, ${ }^{2}$ weak basal lower oesophageal sphincter pressure, $^{3}$ and transient lower oesophageal sphincter relaxations. ${ }^{3}$ Some, possibly even all, of these factors play a role, their relative contributions varying from patient to patient and within a single patient. For instance, basal lower oesophageal sphincter pressure can vary over several hours from barely recordable to a very hearty value. ${ }^{4}$ We should recognise that reflux episodes nearly always result from a combination of factors. In particular, it seems likely that hiatus hernia could increase the likelihood of occurrence of reflux during a transient lower oesophageal sphincter relaxation.

Mechanisms of gastro-oesophageal reflux have been evaluated by mechanical modelling, sampling of gastrooesophageal junction pressures and by recording of events associated with occurrence of individual gastrooesophageal reflux episodes. The intermittency of reflux shows that the mechanical conditions that cause reflux only occur every now and then. Mechanical modelling and sampling of gastro-oesophageal junction pressure are unlikely to be fruitful, given this complexity and the variability of mechanical events at the gastro-oesophageal junction. Events associated with occurrence of gastrooesophageal reflux episodes can only be evaluated by monitoring of mechanical function simultaneously with monitoring for reflux episodes, usually by $\mathrm{pH}$ monitoring, ${ }^{2}$ but also, in the case of provocative manoeuvres, by fluoroscopy. ${ }^{5}$ This approach has given useful insights into patterns of gastro-oesophageal junction pressure and diaphragmatic hiatal activity associated with reflux episodes.

How then can the insights derived from the study of Kahrilas et al be used to advance understanding of the ways in which hiatus hernia influences gastro-oesophageal competence? This study now allows confident interpretation of two axially separated high pressure zones as being due to the hiatus hernia and the lower oesophageal sphincter. Consequently, in some studies, it is reasonable to dispense with fluoroscopic imaging, and rely on methods that give reliable monitoring of the axial patterning of pressure from below a hiatus hernia into the oesophagus. This will give continuous information about mechanical function across the gastro-oesophageal junction. These data can then be readily correlated with occurrence of reflux, recognised by simultaneous distal oesophageal $\mathrm{pH}$ monitoring. Such studies need a sophisticated approach to monitoring of pressures if they are to give technically adequate insights. Kahrilas et al have shown a complex pattern of change of pressures from below the diaphragm into the distal oesophagus by the use of pull-through during suspended respiration during different patterns of straining and at rest. In order to monitor pressures in this region, the recording assembly will need to be stationed, and to record pressures from as many as 21 recording sites only about 3-5 $\mathrm{mm}$ apart in order to resolve adequately patterns of 
pressure over a critical span of $6-10 \mathrm{~cm}$ despite movements of the gastro-oesophageal junction. Such recordings could be combined with luminal electromyographic monitoring of diaphragmatic hiatal contractions. ${ }^{2}$ Such manometric methodology now exists, and could in the near future be adapted for use in ambulatory studies which could reveal how hiatus hernia influences gastro-oesophageal competence under normal living conditions. If and when you come across such a study in the future, spare a thought for the researchers who will have evaluated up to 23 simultaneously gathered squiggly lines!

J DENT
Royal Adelaide Hospital,

North Terrace Adelaide,

SA 5000, Australia

Email:jdent@gastro.rah.sa.gov.au

1 Ingelfinger FI. Esophageal motility. Physiol Rev 1958;38:533-84.

2 Mittal RK, Balaban DH. The esophagogastric junction. N Engl f Med 1997; 336:924-32.

3 Mittal RK, Holloway RH, Penagini R, et al. Transient lower esophageal sphincter relaxation. Gastroenterology 1995;109:601-10.

4 Dent J, Holloway RH, Toouli J, et al. Mechanisms of lower oesophageal sphincter incompetence in patients with symptomatic gastro-oesophageal reflux. Gut 1988;29:1020-8.

5 Sloan S, Rademaker AW, Kahrilas PJ. Determinants of gastroesophageal junction incompetence: hiatus hernia, lower esophageal sphincter, or both? Am Intern Med 1992;117:977-82.

\section{The quality of quality of life}

Functional bowel disorders are highly prevalent in the general population. In the US Householder Survey about $70 \%$ of respondents had at least one functional bowel disorder, $9.4 \%$ had irritable bowel syndrome (IBS) and $2.9 \%$ had functional dyspepsia. ${ }^{1}$ These disorders often have significant symptom overlap making them difficult to distinguish. ${ }^{23}$ In this issue (see page 527), Chassany et al describe the initial development, preliminary psychometric testing and cross-cultural assessment of a multi-item disease specific health related quality of life (HRQOL) instrument for use in multinational clinical trials of IBS or functional dyspepsia. If only an efficacious therapy could be found!

Health related quality of life assessment is becoming increasingly recognised as an important outcome and predictor for patients with chronic diseases. HRQOL is generally assessed by quantitative questionnaires completed by patients themselves which assess physical and psychosocial attitudes and function. The three kinds of HRQOL measures used in research and practice include global assessments, generic instruments and disease specific instruments. ${ }^{45}$ The global assessment, usually a graded summary such as good, fair or poor or a $10 \mathrm{~cm}$ visual analog scale, may help to predict a relation between simple parameters, like disease severity and function, but is often inadequate for more sophisticated hypothesis testing. Generic instruments are multi-item problem lists that are meant to be independent of sex, age and disease and are applied to compare different populations or diseases. Items are frequently clustered in subscores, such as "physical" or "emotional function", "somatic sensation" or "mental health", or may be summarised into a single score. These can identify unexpected factors affecting HRQOL and may also be useful in predicting health outcome. Disease specific instruments are used to detect important treatment effects or changes with time. They are also multi-item inventories that have been derived in patients with a single condition. Most studies now combine generic and disease specific instruments to optimise the ability to detect important HRQOL changes and to avoid missing unexpected effects. ${ }^{45}$

Before using an HRQOL index, it must undergo psychometric assessment of validity, reliability, and responsiveness. ${ }^{4-7}$ Validity is a comparison of the new index score and a reference score (convergent validity) or a construct of what the new index is measuring, such as a prediction that patients with more severe disease will have poorer HRQOL scores (construct validity). 5 "Reliability" is an assessment of the measurement error of scores or correlations among items or subscores. "Responsiveness" gives a signal-to-noise ratio and allows interpretation of what degree of change is clinically important. ${ }^{8}$

The FDDQL developed by Chassany and colleagues passed through four stages: item selection, test-retest reliability evaluation, therapeutic trials, and cross-cultural validation (French, English, German). The final questionnaire of 43 items has responses graded on a five point scale and aggregated into eight subscales [daily activities, anxiety, diet, sleep, discomfort, coping with disease, control of disease, stress], seven of these summed in a global score (excluding stress). Scores range from 0 to 100, with a higher score representing better HRQOL. Validity was confirmed by showing that:

- Mean scores in functional dyspepsia or IBS were similar among countries. Spearman correlations of FDDQL and SF-36 subscales ranged from 0.41 to 0.69 (convergent validity).

- Mean scores, stratified by the number of gut symptoms present (discriminant validity), gave progressively lower scores.

- Item to subscale Pearson correlations (structure confirmation) were $r>0.4$.

- Mean scores among five subgroups (graded by the investigator from no handicap to severe handicap) demonstrated increasingly poor HRQOL.

Test-retest reliability of the final instrument will need to be re-evaluated as there have been three iterations since the first questionnaire. Internal consistency, as measured by Cronbach's alpha of 0.94 , was excellent. Sensitivity to detect change over time was not assessed and will need to be undertaken during a clinical trial.

Since this study was initiated, two other disease specific IBS HRQOL instruments have been described. ${ }^{89}$ The IBSQOL of Hahn et al has 30 items, nine subscales, with responses of five or six grades, each score of 0 to 100 (higher score better HRQOL). ${ }^{8}$ Scores could significantly distinguish patients with non-IBS gut disorders from two referred groups with IBS and internal consistency was as good as the FDDQL with alphas of 0.66 to 0.93 . The IBSQOL, the second questionnaire (note the hyphen), is probably the most extensively validated of the three instruments. ${ }^{9}$ This index has 34 items, eight subscales, item responses are graded on a five point scale, total score 0 to 100 with a higher score indicating poorer HRQOL. Internal consistency was 0.74 to 0.95 and test-retest reliability intraclass correlations were 0.65 to 0.89 . Comparisons with other instruments (subscales of SF-36, Psychological General Well Being Index, SCL90-R) gave moderate $r$ values of 0.30 to 0.47 , supporting the convergent validity. As the frequency and severity of symptoms increased, IBS- 
QOL scores deteriorated significantly. This instrument, in contrast to the FDDQL, did correlate moderately with pain and also with absenteeism from work. The IBS-QOL has also undergone some cross-cultural validation.

None of these three instruments has yet been assessed for ability to reflect important clinical changes (responsiveness). There is an unsurprising degree of similarity in questions, responses, subscales, and scoring techniques. Even the consistency, reliability coefficients and validation correlations are of a similar magnitude. The FDDQL is somewhat different from the other two IBS indexes in that it seems to incorporate some problems specific to functional dyspepsia and with further validation it will become clear how valuable it can be for this disorder.

The appropriate clinical trial is needed not only for clinicians who wish to improve the HRQOL of these patients, but also to assess the value of these instruments. In such a clinical trial, it will be important to study only one functional bowel disorder, IBS, and other outcomes will be essential, such as disease severity scores, pathophysiology and mechanism of treatment.

Both generic and disease specific HRQOL instruments should be combined to determine which are clinically important changes and to avoid missing unexpected associations. Adjustment of scores should be undertaken for disease severity, and sex and age differences should be explored further. The authors might also consider the study by Eypasch et al who devised a general Gastrointestinal Quality of Life Index (GIQLI), with a group of core items and additional modules for more specific diseases. ${ }^{10}$ Their final questionnaire has five domains, 32 items, graded responses ( 0 to 4 ) and a sumscore of 0 to 128 (a higher score denoting better HRQOL). It was tested for face validity and reliability (ICC 0.92 ), and was shown to be responsive in 194 patients who had laparoscopic cholecystectomy. Scores improved significantly $(\mathrm{p}<0.001)$ from a mean (SD) of $87.3(17.25)$ to 104.5 (17.52) two weeks after surgery. Would it not be wise to consider collaborative efforts among international groups, to expedite full validation of instruments, avoid redundancy and develop more instruments with broader applications for which crosscultural validation could be hastened. A plea for HQQLI (High quality quality of life instruments).

E J IRVINE

Professor of Medicine,

Division of Gastroenterology,

McMaster University,

Hamilton,

Ontario L8N 3Z5, Canada

1 Drossman DA, Li Z, Andruzzi E, et al. U.S. householder survey of functional gastrointestinal disorders. Dig Dis Sci 1993;38:1569-80.

2 Talley NJ, Colin-Jones D, Koch K, et al. Functional gastrointestinal disorders. In: Drossman DA, Richter JE, Talley NJ, et al, eds. The functional disorders. In: Drossman DA, Richter JE, Talley NJ, et al, eds. The function

3 Thompson WG, Creed F, Drossman DA, et al. Functional bowel disorders and functional abdominal pain. In: Drossman DA, Richter JE, Talley NJ, et al, eds. The functional gastrointestinal disorders. Boston: Little Brown and Co., 1994:115-73.

4 Guyatt GH, Feeny DH, Patrick DL. Measuring health related quality of life in chronic disease. Ann Intern Med 1993;118:622-9.

Patrick DL, Deyo RA. Generic and disease-specific measures in assessing health status and quality of life. Med Care 1989;27(suppl):S217-32.

6 Deyo RA, Diehr P, Patrick DL. Reproducability and responsiveness of health status measures: statistics and strategies for evaluation. Control Clin Trials 1991;12:142-58S.

7 Guyatt GH, Walter S, Norman G. Measuring change over time: Assessing the usefulness of evaluative instruments. F Chron Dis 1987;40:171-8.

8 Hahn BA, Kirchdoerfer LJ, Fullerton S, et al. Patient-perceived severity of irritable bowel syndrome in relation to symptoms, health-care utilization and quality of life. Aliment Pharmacol Ther 1997;11:553-9.

9 Patrick DL, Drossman DA, Frederick IO, et al. Quality of life in persons with irritable bowel syndrome. Development and validation of a new measure. irritable bowel syndrome. Devel

10 Eypasch E, Williams JI, Wood-dauphinee S, et al. Gastrointestinal quality of life index: development, validation and application of a new instrument. $\mathrm{Br}$ f Surg 1995;82:216-22.

\section{New kids on the block: pancreatic stellate cells enter the fibrogenesis world}

Two recently published papers have shown that a cell type with morphological features of "vitamin A storing" stellate cells is present in rat and human pancreatic tissue. ${ }^{12}$ These observations add to previous reports addressing the same issue in several other extrahepatic organs, including the lung, the intestine, and the kidney. ${ }^{34}$

Over the past 10 years, a great deal of scientific information has followed the identification of stellate cells as the main cell type involved in the progression of liver fibrosis following chronic tissue damage. ${ }^{5}$ Several roles for this cell type in normal and diseased liver have been highlighted. In addition to storage of vitamin A, these include: (1) excessive deposition of extracellular matrix on activation; (2) modulation of sinusoidal tone in normal liver as a result of their contractile features and possible involvement in the progression of portal hypertension; (3) synthesis of soluble factors affecting liver regeneration (hepatocyte growth factor) or recruitment and differentiation of inflammatory cells; and (4) a possible role in the development of primary and metastatic liver cancer stroma. Most experimental evidence, originally produced in vitro, has subsequently been corroborated by extensive studies in animal models and in tissue sections obtained from patients with different degrees of necroinflammatory and fibrogenic activity. More recently, a consistent number of studies have focused on the intracellular signalling events related to hepatic stellate cell activation, proliferation, migration, synthesis of the extracellular matrix (ECM), and adhesion. The results of these studies have opened new perspectives in the development of pharmacological strategies aimed at modulating the fibrogenic process within the liver. Along these lines, after the initial identification and characterisation of pancreatic stellate cells, the work by Apte and coworkers (see page 534) has been directed at verifying whether these cells, maintained in culture, produce collagen and other ECM components and respond to profibrogenic growth factors such as platelet derived growth factor (PDGF) and transforming growth factor (TGF) $\beta 1$, similarly to their hepatic counterparts. ${ }^{6}$ The results of the study support the concept that pancreatic stellate cells may play a key role in the development of pancreatic fibrosis and open new perspectives in this specific area of research. Recent preliminary data indicate that increased formation of lipid peroxidation products, particularly reactive aldehydes, able to form adducts with acinar cell proteins occurs in one of the most common clinical conditions associated with pancreatic fibrosis, namely chronic alcoholic pancreatitis. ${ }^{7}$ Studies performed in human hepatic stellate cells have shown that reactive aldehydes such as 4-hydroxy-2, 3-nonenal and other 4-hydroxy-2, 3-alkenals of different chain lengths are able to stimulate procollagen type I synthesis and secretion at concentrations which are compatible with a mild to moderate oxidative stress (i.e. lipid peroxidation) occurring in vivo. ${ }^{8}$ It must be stressed that the profibrogenic effects of 
these products, derived from the lipoperoxidative damage of membrane phospholipids, are independent of the more articulated mechanisms involved in chronic tissue repair (tissue damage $>$ inflammatory infiltration $>$ release of profibrogenic growth factors). Along these lines, it would be important to evaluate the role of these compounds in periacinar stellate cell activation and ECM synthesis in future studies. Beyond this important advancement there are other relevant considerations. As already proposed, the presence of stellate cells in several organs and tissues raises the possibility of a "vitamin A storing system". In addition, similarly to hepatic stellate cells, stellate cells in extrahepatic tissues are distributed with specific interstitial features, namely their cytoplasmic projections encircle sinusoidal-like vascular structures. It is therefore possible that stellate cells constitute a class of organ specific pericytes with similar physiological and pathophysiological implications. In this context, a key current issue concerns the origin of this cell type. Because these cells express desmin and smooth muscle $\alpha$-actin, they were assumed to be of myogenic origin. This hypothesis became doubtful when it was reported that stellate cells also express glial fibrillary acidic protein (GFAP) and neural cell adhesion molecule (NCAM). Moreover, recent work by Niki and coworkers has demonstrated that activated hepatic stellate cells express nestin, a class VI intermediate filament protein originally identified as a marker for neural stem cells. ${ }^{10}$ It is generally accepted that cephalic neural crest cells give rise to a variety of cell types, including neurones, glial cells, cartilage, bone, and smooth muscle cells. Consequently, the key question is whether there a common precursor cell somewhere in the neural crest that gives rise to all "stellate cells". It is obvious that further studies on the embryonic origin of these cells are required.

Researchers in the field of hepatic stellate cells are not alone anymore. There are new kids on the block. Good luck!

Dipartimento di Medicina Interna,

Universita degli Studi di Firenze,

Viale G.B. Morgagni, 85,

I-50134 Firenze, Italy

Email:m.pinzani@dfc.unifi.it

1 Apte MV, Haber PS, Applegate TL, et al. Periacinar stellate shaped cells in rat pancreas: identification, isolation, and culture. Gut 1998;43:128-33.

2 Bachem MG, Schneider E, Gross H, et al. Identification, culture, and characterization of pancreatic stellate cells in rats and humans. Gastroenterology

3 Blomhoff R, Wake K. Perisinusoidal stellate cells of the liver: important roles in retinol metabolism and fibrosis. FASEB $\mathcal{F} 1991 ; 5: 271-7$

4 Nagy NE, Holven KB, Roos N, et al. Storage of vitamin A in extrahepatic stellate cells in normal rats. F Lipid Res 1997;38:645-58.

5 Pinzani M. Novel insights into the biology and physiology of the Ito cell. Pharmac Ther 1995;66:387-412.

6 Pinzani M, Gesualdo L, Sabbah GM, et al. Effects of platelet-derived growth factor and other polypeptide mitogens on DNA synthesis and growth of cultured liver fat-storing cells. F Clin Invest 1989;84:1786-93.

7 Galli A, Pignalosa P, Grappone C, et al. Enhanced lipid peroxidationderived aldehydes in chronic pancreatitis suggest a role in its pathogenesis. derived aldehydes in chronic pancreatitis suggest a
Ital f Gastroenterol Hepatol 1998;30(suppl 2):A147.

8 Parola M, Pinzani M, Casini A, et al. Induction of procollagen type I expresParola $M$, Pinzani $M$, Casini A, et al. Induction of procollagen type I expres-
sion and synthesis in human hepatic stellate cells by 4-hydroxy-2, 3-nonenal and the other 4-hydroxy-2, 3-alkenals is related to their molecular structure. Biochem Biophys Res Commun 1996;222:261-4

9 Yamada E, Hirosawa K. The possible existence of a vitamin A-storing cell system. Cell Struct Funct 1976;1:201-4.

10 Niki T, Pekny M, Hellemans K, et al. Class VI intermediate filament protein nestin is induced during activation of rat hepatic stellate cells. Hepatology (in press)

See article on page 568

\section{Budd-Chiari syndrome: blocked veins, open mind}

The article by Fisher et al (see page 568) on the various techniques that may be used in the management of BuddChiari syndrome is timely, and demonstrates some important points. This condition must be considered as part of the differential diagnosis of ascites in all patients, though the mode of presentation may be protean and hence may trap the unwary. The classic syndrome of ascites, hepatomegaly and abdominal pain occurs with obstruction of hepatic venous outflow at any level-from the hepatic venules (e.g. veno-occlusive disease) to the suprahepatic vena cava. Constrictive pericarditis may also mimic the presentation. The syndrome may run a number of clinical courses which also partly determine treatment. The applicability of any treatment is also influenced by the fact that the inferior vena cava may be blocked in $20 \%$ of cases, and portal vein thrombosis evident in $10 \%$.

The patient may present with fulminant hepatic failure, with notable coagulopathy, encephalopathy and renal impairment. A more indolent presentation is also well recognised, with ascites with a high protein content, mildly impaired hepatic function, and a variable degree of fibrosis on biopsy. Finally, well compensated patients with hepatic vein obstruction and no ascites have been described; the development of numerous intrahepatic collaterals with time is said to account for this difference. ${ }^{1}$ A thrombophilic disorder will be found in up to $75 \%$ of patients. This may have additional implications with respect to family screening. Primary myeloproliferative disorders are the most common, but all of the inherited prothrombotic tendencies have been shown to be causal, particularly in association with the oral contraceptive pill and the puerperium. Paroxysmal nocturnal haemoglobinuria, lupus anticoagulant, Behçet's syndrome, vasculitides, ulcerative colitis, and mechanical obstruction of the hepatic veins by tumour or cysts are also possible associations or causes. Not uncommonly, two or more prothrombotic tendencies may co-exist, and thus bone marrow biopsy is mandatory.

Having considered the diagnosis, and when initial imaging suggests abnormal venous outflow, hepatic venography and visualisation of the inferior vena cava with pressure measurements above and below the liver is the next step, as outlined by Fisher and colleagues. This will confirm:

- The site of the hepatic vein blockage. Short segment hepatic vein stenosis/occlusion may be amenable to local angioplasty and stenting.

- Whether there is significant caval compression secondary to caudate lobe hypertrophy. If present, a standard portocaval (infrahepatic) shunt will not reduce hepatic congestion, and a mesoatrial (suprahepatic) shunt is required, unless the cava is stented beforehand.

- Liver histology. A transjugular liver biopsy should be performed and will establish the degree of fibrosis. In our experience, it is rare that this is technically unsuccessful, even with extensive proximal hepatic vein occlusion. A small "beak" arising from the inferior vena cava may indicate hepatic vein origin, and with care a biopsy can be performed as the cava is within the liver. At this point our experience differs from that of the Birmingham group. We do not see short segment hepatic vein occlusion. Caval webs are well described in Japan and South Africa, but the authors comment that most of their patients were from Western Europe. We are unable to explain this anomaly. 
Clearly a focal occlusion may be amenable to angioplasty and stenting, and the authors document a good technical and overall success rate, and this technique can be added to the other various treatments used in this syndrome. There are, however, some important caveats. The right and middle hepatic veins are relatively easy to visualise at hepatic venography, whereas the left hepatic vein may be difficult to gain access to. Standard teaching advises that all of the liver should be decompressed and one could envisage that left hepatic vein occlusions could be missed, unless very proximal. In this respect, wedge pressure measurements are an important indicator of therapeutic success. As Budd-Chiari syndrome is a postsinusoidal condition, a successful shunt should reduce the wedged hepatic venous gradient to less than $6 \mathrm{~mm} \mathrm{Hg}$. In the paper by Fisher et al, only seven of 21 patients had data on wedge pressure responses, and gradients were not given. Theoretically, incomplete restoration of normal hepatic venous blood flow, as reflected by failure to normalise wedge pressure, could allow the fibrotic process to continue. Moreover, using the technique of percutaneous hepatic vein angioplasty, the patients require, according to the Birmingham protocol, annual follow up venography, and to compare their results with the group of patients who had surgical shunts may not be entirely valid as, by their own admission, these patients had more proximal occlusion and therefore perhaps greater overall intrahepatic thrombosis.

None the less, the paper demonstrates the heterogeneity of this patient population and some of the difficulties encountered, as well as a sensible clinical algorithm, and the potential clinical role of a relatively non-invasive radiological technique. For patients with a fulminant presentation, hepatic transplantation is the treatment of choice, and overall success rates of $70 \%$ have been recorded. ${ }^{23}$ For those patients without fulminant failure, treatment will be influenced by the presence of fibrosis on liver biopsy, and the length of history. Text books and several papers ${ }^{45}$ suggest that if there is an acute presentation (history less than 16 weeks), with little fibrosis, no portal vein thrombosis or significant caval gradient, then a side to side portocaval shunt is the treatment of choice, with a five year survival of $95 \%$ in one series. ${ }^{5}$ One could argue that the Birmingham results should be viewed in this context. However, these patients may be more fragile than the textbooks would have one believe (for some, the procoagulant process will have been present since birth) and acute liver failure may be precipitated by shunting, despite the correct investigational pathway. This has been the experience of ourselves, ${ }^{6}$ and others. Ringe et al performed four emergency transplants on four patients with Budd-Chiari syndrome shunted 11-22 days previously, in whom shunt induced hepatic failure developed. ${ }^{3}$ In this respect transjugular intrahepatic portosystemic stent shunt is an attractive proposition - it bypasses the caval obstruction and does not compromise the surgical field should transplantation be necessary. ${ }^{78}$ Whichever mode of treatment is chosen, it is our opinion that these patients should be referred to a transplant unit as hepatic decompensation may not be predictable.

The final point, well made by Fisher et al, is the need for life long, full anticoagulation. Mesh metal stents notoriously block in patients with cirrhosis - this occlusion rate may well be higher in patients who have a prothrombotic state.

D PATCH

Liver Unit, A BURROUGHS

Royal Free Hospital School of Medicine,

Pond Street,

London NW3 2QG, UK

1 Hadengue A, Poliquin M, Vilgrain V, et al. The changing scene of hepatic vein thrombosis: recognition of asymptomatic cases. Gastroenterology 1994;106:1042-7.

2 Halff G, Todo S, Tzakis AG, et al. Liver transplantation for the management of Budd-Chiari syndrome. Ann Surg 1990;211:43-9.

3 Ringe $\mathrm{B}$, Lang $\mathrm{H}$, Oldhafer $\mathrm{K}-\mathrm{J}$, et al. Which is the best surgery for Budd-Chiari syndrome: venous decompression or liver transplantation. A single centre experience with 50 patients. World f Surg 1994;21:233-9.

4 Bismuth H, Sherlock, D. Portasystemic shunting versus liver transplantation for the Budd-Chiari syndrome. Ann Surg 1991;214:581-9.

5 Orloff MJ, Orloff MS, Daily PO. Long term treatment of Budd Chiari syndrome with portal decompression. Arch Surg 1992;127:1182-8.

6 Thompson NP, Miller AD, Hamilton G, et al. Emergency rescue hepatic Thompson NP, Miller AD, Hamilton $\mathrm{G}$, et al. Emergency rescue hepatic
transplantation following shunt surgery for Budd-Chiari syndrome. Eur $\mathcal{F}$ transplantation following shunt surger
Gastroenterol Hepatol 1994;6:835-7.

7 Ochs A, Sellinger M, Haag K, et al. Transjugular intrahepatic portosystemic stent-shunt (TIPS) in the treatment of Budd-Chiari syndrome. $₹$ Hepatol 1993;18:217-25.

8 Strunk H, Textor J, Brensing KA, et al. Acute Budd-Chiari syndrome: treatment with transjugular intrahepatic portosystemic shunt. Cardiovasc Intervent Radiol 1997;20:311-13. 